(2) Open Access Full Text Article

\title{
Finasteride nano-transferosomal gel formula for management of androgenetic alopecia: ex vivo investigational approach
}

This article was published in the following Dove Press journal:

Drug Design, Development and Therapy

\author{
Osama AA Ahmed ${ }^{1,2}$ \\ Waleed Y Rizq' \\ 'Department of Pharmaceutics, \\ Faculty of Pharmacy, King Abdulaziz \\ University, Jeddah, Saudi Arabia; \\ ${ }^{2}$ Department of Pharmaceutics and \\ Industrial Pharmacy, Faculty \\ of Pharmacy, Minia University, \\ Minia, Egypt
}

\begin{abstract}
Introduction: Finasteride (FIN) is known as type II $5 \alpha$-reductase inhibitor, which has been approved for the treatment and prevention of androgenetic alopecia. Administration of FIN by oral route has led to undesirable systemic side effects that include mood disturbance, gynecomastia, decreased libido, erectile dysfunction, and ejaculation disorder. The aim was to improve FIN delivery through skin layers and hair follicles that could possibly reduce its major side effects resulting from long-term oral administration for the treatment and prevention of male pattern baldness.
\end{abstract}

Materials and methods: FIN was formulated as nano-transferosomal (NTF) gel formulations (F1-3). The prepared formulations were characterized for encapsulation efficiency, particle size, ex vivo skin permeation, and kinetic modeling. In addition, visualization of NTF skin penetration using a fluorescence laser microscope was carried out for the selected formula (F2).

Results and discussion: The results showed that FIN encapsulation efficiency percentage was $69.72 \pm 8.36,89.43 \pm 6.82$, and $93.1 \pm 1.93$ for F1, F2, and F3, respectively. FIN-NTF average vesicle sizes were $299.6 \pm 45.6,171 \pm 25.6$, and $197.4 \pm 29.1 \mathrm{~nm}$ for F1, F2, and F3, respectively. FIN-NTF formulations (F1-3) showed enhancement and improvement in the amount of FIN permeated compared with raw FIN gel formula. The NTF formula revealed uniform fluorescence (rhodamine) intensity across rat skin, which indicated improved delivery through skin layers compared with control gel formula.

Conclusion: These results indicated that NTF gel formula showed the ability to boost FIN delivery across skin layers and could be applied as an alternative for oral therapy.

Keywords: nano-lipid carriers, $5 \alpha$-reductase inhibitor, hair loss, male-pattern baldness

\section{Introduction}

Finasteride (FIN, Figure 1) is the first specific competitive inhibitor of type II $5 \alpha$-reductase inhibitor that is used for the treatment of human benign prostatic hyperplasia (BPH) and androgenetic alopecia. ${ }^{1-3}$ FIN is classified in the Biopharmaceutical Classification System as class II drug possessing high permeability and low solubility. The low solubility of FIN affects the process of drug dissolution and the rate and extent of FIN bioavailability. FIN was approved by the US Food and Drug Administration (FDA) for the treatment of BPH in 1992 and male pattern baldness (male pattern hair loss) in 1997. FIN is available in two different dosage forms. The dosage of $1 \mathrm{mg}$ /day is utilized for the treatment of men with male pattern hair loss, ${ }^{4-6}$ while $5 \mathrm{mg}$ /day dosage is used for the relief of symptoms associated with, and the prevention of disease progression of, BPH. ${ }^{5}$
Correspondence: Osama AA Ahmed Department of Pharmaceutics and Industrial Pharmacy, Faculty of Pharmacy, King Abdulaziz University, PO Box 80260, Jeddah 21589, Saudi Arabia Email oaahmed@kau.edu.sa 


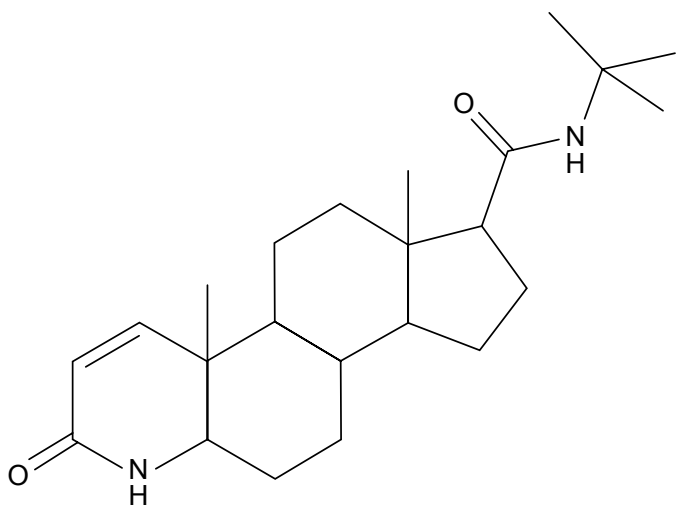

Figure I Chemical structure of finasteride.

FIN reduces androgenetic alopecia through the specific and competitive inhibition of $5 \alpha$-reductase enzyme. This enzyme is responsible for the dihydrotestosterone (DHT) production in hair follicles. Accordingly, FIN decreases the DHT levels in the hair follicles and in the blood. ${ }^{7}$ Although the FDA has approved FIN to use in oral formulations, the administration of FIN by this route has led to undesirable systemic side effects. ${ }^{8,9}$ The undesirable effects include mood disturbance, gynecomastia, decreased libido, erectile dysfunction, and ejaculation disorder. ${ }^{10,11}$ Consequently, successful topical administration of FIN could improve FIN penetration and increase its accumulation in the skin layers and hair follicles and could possibly reduce its major side effects resulting from oral administration. ${ }^{12-14}$

Topical liposomal formulations have been used widely for their safety, sustained release property, and improved clinical efficacy. ${ }^{15}$ However, the draw backs of utilizing conventional liposomes in transdermal delivery, as they are trapped in the upper skin layers, have been previously reported. ${ }^{16,17}$ Nano-transferosomes (NTF) are one of the new generations of liposomes which have been used to improve drug delivery. ${ }^{18-21}$ NTF are defined as flexible and ultra-deformable vesicles composed of phospholipids and an edge activator. The edge activator destabilizes the lipid bilayers of the vesicular structure that enhances squeezing and penetration through skin layers. ${ }^{19-22}$ Transdermal drug delivery systems that are composed of NTF have been studied widely. ${ }^{18-25}$ Previous studies have incorporated NTF into gel bases and batch formulations. ${ }^{26,27}$ This work aims for the preparation and evaluation of FIN-NTF gel formula for the treatment of androgenetic alopecia. FIN-NTF formulations were characterized for encapsulation efficiency (EE) and particle size. In addition, ex vivo skin permeation and visualization of FIN-NTF skin penetration using a fluorescence microscope for FIN formulation were carried out to achieve a formulation with potentially improved performance that can avoid side effects resulting from the orally administered dosage forms.

\section{Materials and methods}

FIN was obtained from DEEF Pharmaceutical industries (Badayea, Qassim, KSA). Span 65 and rhodamine were purchased from Sigma-Aldrich Co. (St Louis, MO, USA). Phospholipon 90G was obtained from Thermo Fisher Scientific (Waltham, MA, USA). Methanol was purchased from EMD Millipore (Billerica, MA, USA).

\section{Formulation of FIN-NTF}

FIN-NTF were prepared by modified lipid film hydration technique as previously described. ${ }^{19}$ Briefly, FIN and Phospholipon 90G were mixed in molar ratios of 1:2.0, 1:2.75, and 1:3.5 to obtain formulations F1, F2, and F3, respectively. Span $65,5 \% \mathrm{w} / \mathrm{w}$ of total solid components, was used as edge activator (Table 1). All the three components were dissolved in $50 \mathrm{~mL}$ of methanol and sonicated using a water bath sonicator for 10 minutes. The prepared solution was then evaporated using rotary evaporator at $45^{\circ} \mathrm{C}$ (R-200; BÜCHI Labortechnik AG, Flawil, Switzerland). The formed thin film was kept overnight in vacuum oven for complete removal of solvent. The film was then hydrated using phosphate-buffered saline (PBS), $\mathrm{pH} 7.2$, for 2 hours at $45^{\circ} \mathrm{C}$.

\section{Characterization of the prepared FIN-NTF \\ Determination of FIN entrapment efficiency}

The drug entrapment efficiency percent $(\mathrm{EE} \%)$ was determined using indirect method as described previously. ${ }^{19}$ Aqueous dispersion samples of FIN-NTF formulations were centrifuged at $30,000 \mathrm{rpm}$ for 45 minutes at $4^{\circ} \mathrm{C}$. The supernatant was further filtered through $0.1 \mu \mathrm{m}$ membrane filter. FIN concentration in the supernatant solution (unentrapped FIN)

Table I Composition, particle size, and EE\% of FIN-NTF formulations

\begin{tabular}{llll}
\hline Formulation & FI & F2 & F3 \\
\hline FIN & $100 \mathrm{mg}$ & $100 \mathrm{mg}$ & $100 \mathrm{mg}$ \\
Phospholipon 90G & $405 \mathrm{mg}$ & $559 \mathrm{mg}$ & $710 \mathrm{mg}$ \\
Span 65 & $25 \mathrm{mg}$ & $33 \mathrm{mg}$ & $41 \mathrm{mg}$ \\
\hline Characterization & FI & F2 & F3 \\
\hline Particle size $(\mathrm{nm})^{*}$ & $299.6 \pm 45.6$ & $171 \pm 25.6$ & $197.4 \pm 29.1$ \\
EE (\%)* & $69.72 \pm 8.36$ & $89.43 \pm 6.82$ & $93.1 \pm 1.93$ \\
\hline
\end{tabular}

Note: *Results are presented as mean \pm SD.

Abbreviations: FIN, fnasteride; EE, entrapment efficiency percent; NTF, nanotransferosomes. 
was calculated after analysis utilizing high-performance liquid chromatography (HPLC) assay method as previously reported. ${ }^{28}$ The EE\% was calculated according to Equation (1), where $C_{1}$ is the total amount of FIN included in the preparation of FIN-NTF and $C_{2}$ is the amount of unentrapped FIN:

$$
\mathrm{FIN} \mathrm{EE} \%=\frac{C_{1}-C_{2}}{C_{1}} \times 100
$$

\section{Particle size analysis of the prepared FIN-NTF}

The prepared FIN-NTF were subjected to particle size analysis using Zetatrac (Microtrac ${ }^{\circledR}$ Inc., Montgomeryville, PA, USA) particle size analyzer. The prepared formulations were diluted with distilled water and sonicated for 30 seconds. The average particle size was calculated using five replicate samples.

\section{FIN-NTF transmission electron microscope investigation}

The prepared FIN-NTF (F3) were studied using a transmission electron microscope (TEM) (100CX; JEOL, Tokyo, Japan). A drop of diluted FIN-NTF was added onto a TEM grid and then stained with $2 \%$ uranyl acid. The grid was dried before investigation.

\section{Preparation of FIN-NTF gel formula}

A polymeric solution was prepared by loading FIN-NTF into hydroxypropyl methylcellulose (HPMC). Briefly, FIN-NTF (equivalent to $1 \mathrm{~g}$ FIN) were dispersed in distilled water $(100 \mathrm{~mL})$ and sonicated for 30 seconds to diffuse any lumps in the dispersion. After that, HPMC (2 g) was dissolved in the NTF aqueous dispersion (100 mL), using magnetic stirrer, and stored at $4^{\circ} \mathrm{C}$. In addition, raw FIN gel was prepared by dispersing powdered FIN ( $1 \mathrm{~g}$ ) in aqueous (HPMC 2\% w/v) solution using the same procedure described for the preparation of FIN-NTF gel.

\section{Ex vivo skin permeation of FIN-NTF gel formula}

Permeation studies were carried out using Microette Plus Hanson Automated Vertical Diffusion Cells (Hanson Research, Chatsworth, CA, USA). PBS (pH 5.8) was used as a diffusion medium at $32^{\circ} \mathrm{C} \pm 0.5^{\circ} \mathrm{C}$ and the stirring rate was $400 \mathrm{rpm}$. Male Wistar rat's skin from the abdominal region was shaved by electric clipper, excised $\left(3 \times 3 \mathrm{~cm}^{2}\right)$, and freed from subcutaneous fats. The animals were provided by King Fahd Medical Research Centre, King Abdulaziz University, Jeddah, Saudi Arabia. The Research Ethics Committee of
Faculty of Pharmacy, King Abdulaziz University, approved animal use that followed the Helsinki agreement protocol and the Guiding Principle in Care and Use of Animals (DHEW publication NIH 80-23).

The excised skin parts were examined for skin integrity using a magnifier. The skin was soaked in PBS ( $\mathrm{pH} 5.8$ ) for 3 hours. After that, the prepared skin was mounted between the donor and receptor compartments of the cells. Permeation of FIN-NTF gel was evaluated and amounts of FIN permeated were determined using HPLC. The obtained data were compared with permeation data of raw FIN-loaded HPMC gel.

\section{Analysis of permeation parameters and kinetic modeling}

The release pattern of FIN was determined according to relation of the cumulative amount of FIN permeated (Q) per unit area as a function of time. Steady-state flux $\left(\mathrm{J}_{\mathrm{SS}}\right)$, permeability coefficient (Pc), and diffusion coefficient (D) were calculated as previously reported. ${ }^{26,29}$ In addition, the obtained permeation data were fitted into different kinetic models: zero, first, Higuchi, Hixson-Crowell, and BakerLonsdale models. ${ }^{19,30,31}$ Linearity and correlation coefficient (r) were evaluated.

\section{Visualization of NTF skin penetration using a fluorescence laser microscope}

FIN-NTF gel formulation (F2) was selected for the fluorescence laser microscope investigation. Rhodamine $(0.15 \mu \mathrm{mol} / \mathrm{mL})$ was used instead of FIN in the preparation of NTF gel as described in the previous sections. The rhodamine-loaded NTF were applied on the excised rat skin and penetration of rhodamine-NTF gel formulation across rat skin was investigated using automated diffusion cell sampling system as previously described. For comparative study, gel formula loaded with raw rhodamine (control) was prepared and treated as described earlier for NTF loaded with rhodamine gel formulation. Skin removed after 1 and 5 hours was kept in formalin. Blocks of paraffin wax-embedded skin samples were prepared and cut into $4 \mu \mathrm{m}$ thick longitudinal sections using a microtome (Leica Microsystems SM2400; Cambridge, United Kingdom). The prepared samples were finally observed using Zeiss Axio Observer D1 Inverted Dic Fluorescence microscope (Carl Zeiss AG, Oberkochen, Germany) with a 470/40 nm excitation filter, a 495 beam splitter, and a 525/50 nm emission filter. Images were acquired with identical acquisition parameters, with minimum excitation and gain. 


\section{Results and discussion}

Formulation and characterization of

\section{FIN-NTF}

FIN-NTF formulations were prepared based on a previously reported study. ${ }^{19}$ The prepared formulations were obtained through preparation of drug-to-phospholipid molar ratios of $1: 2.0,1: 2.75$, and $1: 3.5$, and surfactant hydrophile-lipophile balance (HLB) value of 2.08 (Table 1). FIN EE\% in the prepared NTF was found to be $69.72 \pm 8.36,89.43 \pm 6.82$, and $93.1 \pm 1.93$ for F1, F2, and F3, respectively (Table 1). Formula F1 showed the lowest EE\% because of the low FIN: phospholipid lipid ratio (1:2) that could lead to the inability of NTF components to fully entrap FIN amount included in the formula preparation. The high $\mathrm{EE} \%$ observed for the prepared F2 and F3 formulations could be related to the low aqueous solubility of FIN that favors the entrapment of FIN in the NTF vesicles. The high EE\% results obtained for F2 and F3 formulations are in agreement with the previously reported results for the entrapment of sildenafil in NTF. ${ }^{19}$

The prepared NTF formulations showed average particle size of $299.6 \pm 45.6,171 \pm 25.6$, and $197.4 \pm 29.1 \mathrm{~nm}$ for F1, F2, and F3, respectively (Table 1). The reduced vesicle size of the prepared NTF is attributed to the hydrophobic nature, ie, low HLB of the surfactant. This finding is attributed to the limited water uptake into vesicle core and the reduced surface energy associated with increased hydrophobicity of edge activator that leads to formation of smaller vesicles. ${ }^{32}$ TEM images of the prepared FIN-NTF vesicles (Figure 2) showed vesicles with spherical shape and comparable average vesicle

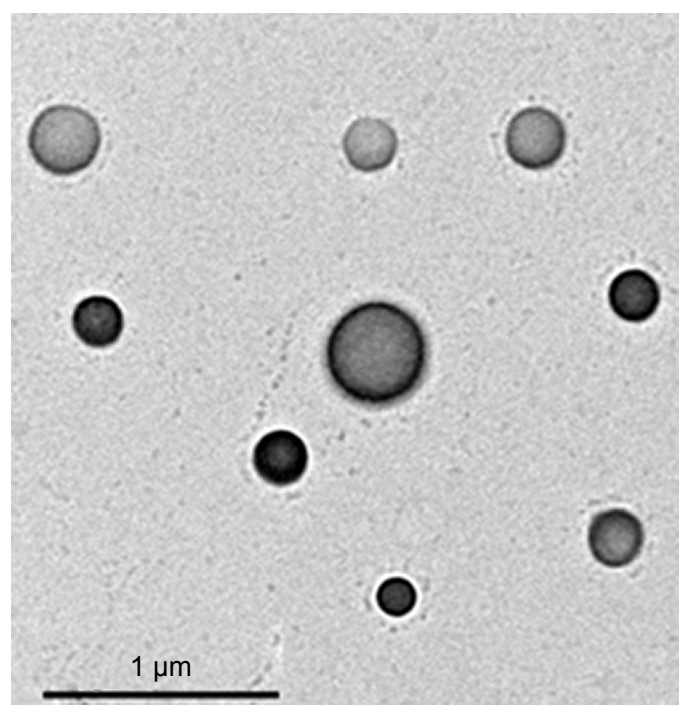

Figure 2 TEM images of the prepared FIN-NTF vesicles (F3).

Abbreviations: FIN, finasteride; NTF, nano-transferosomes; TEM, transmission electron microscopy. size range similar to the data obtained by using Zetatrac particle size analyzer.

\section{Ex vivo skin permeation of FIN-NTF gel formula}

Ex vivo investigation of FIN-NTF formulations (F1-3) injected through excised rat skin was carried out as an indication to the expected in vivo performance. The data regarding cumulative amount of FIN permeated from NTF gel formulations were compared with raw FIN-loaded gel formula (control gel), as shown in Figure 3. The results revealed a significant enhancement and improvement $(p<0.05)$ in the amount of FIN permeated compared with raw FIN gel formula. FIN-NTF gel formulations (F1-3) showed a sustained release pattern of FIN over the 24-hour study period. However, raw FIN gel formula showed slower permeation pattern with the maximum amount of FIN permeated after 24 hours being $13.6 \pm 3.61 \mu \mathrm{g}$ compared with 66.03 , 48.98, and $41.81 \mu \mathrm{g}$ of FIN permeated from FIN-NTF gel formulations F1, F2, and F3, respectively. This could be attributed to the improved nano-dispersion of FIN in the NTF and the ability of ultra-deformable NTF vesicles to deliver FIN through skin layers. ${ }^{32}$

\section{Analysis of permeation parameters and kinetic modeling}

The calculated FIN skin permeation parameters revealed improved results of steady-state flux $\left(\mathrm{J}_{\mathrm{SS}}\right)$, permeability coefficient (Pc), and diffusion coefficient (D) for FIN-NTF gel formulations (F1-3) compared with raw FIN gel (Table 2). FIN-NTF gel formula improved permeation parameters compared with raw FIN gel (control), which could be attributed to the flexibility and deformable structure of transferosomes that

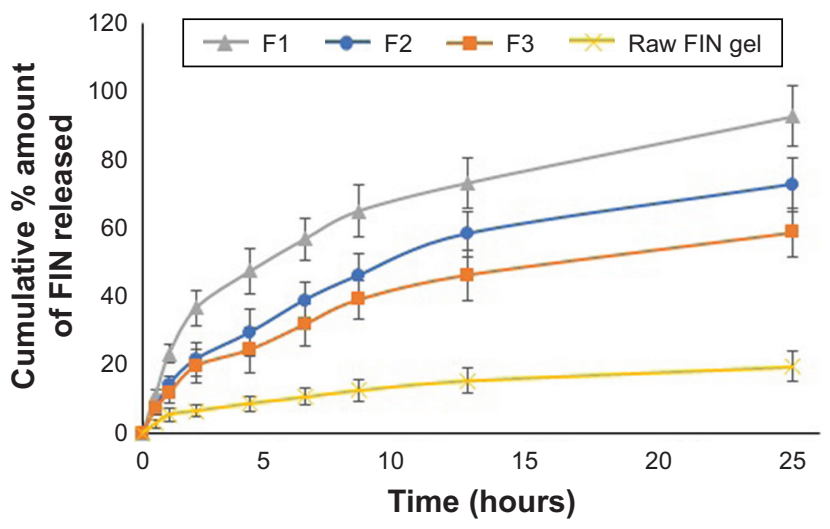

Figure 3 Ex vivo cumulative amount of FIN permeate from rat skin after application of FIN-NTF gel formula and raw FIN gel formula.

Abbreviations: FIN, finasteride; NTF, nano-transferosomes. 
Table 2 Ex vivo permeation parameters for FIN-NTF gel formulations (FI-3) and raw FIN gel

\begin{tabular}{lllll}
\hline Formulation & $\begin{array}{l}\boldsymbol{D}_{\max } \\
(\boldsymbol{\mu g}) \pm \mathbf{S D}\end{array}$ & $\begin{array}{l}J_{\text {ss }} \\
\left(\mu \mathbf{g} / \mathbf{c m}^{2} \cdot \mathbf{h}\right)\end{array}$ & $\begin{array}{l}\mathbf{P c} \\
(\mathbf{c m} / \mathbf{h}) \times 1 \mathbf{0}^{-2}\end{array}$ & $\begin{array}{l}\mathbf{D} \\
\left(\mathbf{c m}^{2} / \mathbf{h}\right) \times 1 \mathbf{0}^{-2}\end{array}$ \\
\hline Raw FIN gel & $13.95 \pm 3.4$ & 0.368 & 0.516 & 0.108 \\
FI & $66.03 \pm 7.9$ & 1.504 & 2.112 & 1.815 \\
F2 & $48.98 \pm 9.3$ & 1.288 & 1.808 & 1.35 \\
F3 & $41.81 \pm 4.3$ & 1.919 & 2.693 & 1.77 \\
\hline
\end{tabular}

Abbreviations: FIN, finasteride; NTF, nano-transferosomes; $D_{\max }$, maximum amount of drug permeated; $J_{s s}$ steady-state flux; Pc, permeability coefficient; $D$, diffusion coefficient.

facilitate their penetration through skin layers. ${ }^{17-20,33}$ Moreover, the presence of edge activator in transferosome formulation destabilizes the lipid bilayer that contributes, along with the nano-size range of transferosomes, to the permeation enhancement of FIN from NTF formula. ${ }^{22,23,33}$ The large surface area of the formed FIN-NTF vesicles improves corneocyte contact which, in turn, improves the amount of FIN permeating the skin. ${ }^{19}$ The improved permeation parameters (Table 2) of the FIN-NTF gel formulations indicate the potential application of FIN for improved delivery through skin layers.

Kinetic analysis of the permeation data for FIN-NTF gel formulations (F1-3) and raw FIN gel are presented in Table 3. The results revealed that the permeation of FIN from FIN-NTF gel formulations (F1-3) followed Baker-Lonsdale model as indicated by the highest correlation coefficient $(r)$ of the model (Table 3). However, raw FIN gel followed Higuchi diffusion model (Table 3). Baker-Lonsdale model, derived from Higuchi diffusion model, explains drug release from spherical matrix. ${ }^{31}$

The results of this study for FIN-NTF formulations (F1-3) revealed no significant difference $(p<0.05)$ in particle size, EE\%, and permeation results for F2 and F3. However, both formulations (F2 and F3) showed significant $(p<0.05)$ improvement in particle size and $\mathrm{EE} \%$ results compared with F1 formula. F1 showed improved permeation characters compared with F2 and F3 formulations. The prepared formulations (F1-3) followed Baker-Lonsdale kinetic model. Accordingly, NTF gel formulation (F2) was selected as a model NTF formula for the visualization of skin penetration investigation.

\section{Visualization of NTF skin penetration using a fluorescence laser microscope}

The penetration of fluorescence (rhodamine)-loaded NTF (F2) gel through rat skin layers was compared with raw rhodamine (control) gel, as shown in Figure 4. Results revealed that NTF gel formula improved the delivery of rhodamine through skin layers when compared with control gel formula. The improved delivery of rhodamine using NTF gel formula is indicated by the distribution of fluorescence with uniform intensity through skin layers (Figure 4 [5 h]). Accordingly, NTF gel formula showed the capability to enhance FIN skin penetration into the deeper dermal tissue.

The flexibility and ultra-deformable nature of NTF is responsible for the improved penetration of FIN into skin layers. The edge activator in NTF vesicles imparts flexibility to the vesicular structure, as it destabilizes the lipid bilayers and enhances its squeezing and penetration through the skin. ${ }^{19,33}$ The improved FIN delivery to the entire depth of the skin revealed that improved amounts of FIN are delivered to the scalp skin, keeping FIN levels in serum at low level. Consequently, it allows to reduce scalp skin DHT levels during the long term of course treatment without initiating the undesirable side effects of FIN resulting from chronic high FIN plasma levels taken by other routes of administration.

\section{Conclusion}

FIN-NTF gel formulations have been investigated as possible transdermal delivery systems for FIN. The NTF formulations showed high EE\% and vesicular sizes of 171-299.6 nm. TEM images of the prepared FIN-NTF vesicles showed vesicles

Table 3 Kinetic analysis of permeation data for FIN-NTF gel formulations (FI-3) and raw FIN gel

\begin{tabular}{|c|c|c|c|c|c|}
\hline \multirow[t]{2}{*}{ Formulation } & \multicolumn{5}{|c|}{ Correlation coefficient $(r)$} \\
\hline & Zero & First & Higuchi & Hixson-Crowell & Baker-Lonsdale \\
\hline Raw FIN gel & 0.93823 & -0.94681 & 0.99422 & 0.95342 & 0.99419 \\
\hline $\mathrm{FI}$ & 0.90984 & -0.99616 & 0.98127 & 0.98254 & 0.99925 \\
\hline F2 & 0.94025 & -0.9849 & 0.99266 & 0.97305 & 0.99635 \\
\hline F3 & 0.94062 & -0.97375 & 0.99242 & 0.96401 & 0.99580 \\
\hline
\end{tabular}

Abbreviations: FIN, finasteride; NTF, nano-transferosomes. 
FIN-NTF gel formula

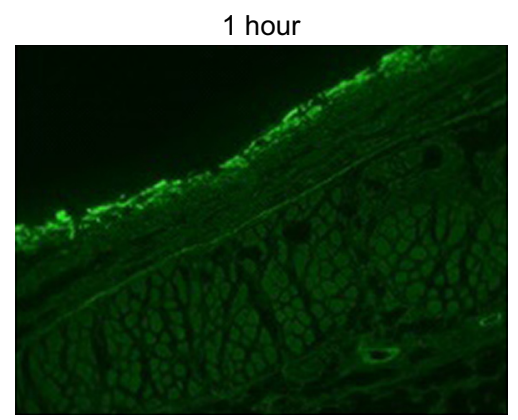

5 hours

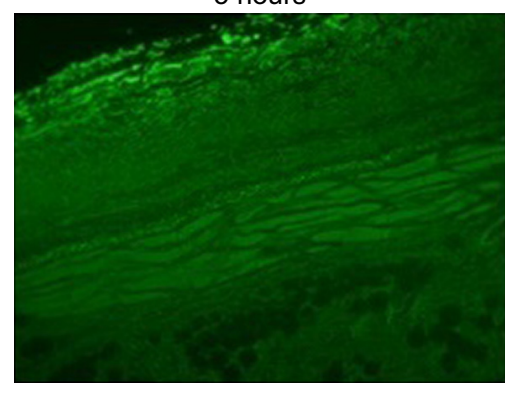

Control gel formula

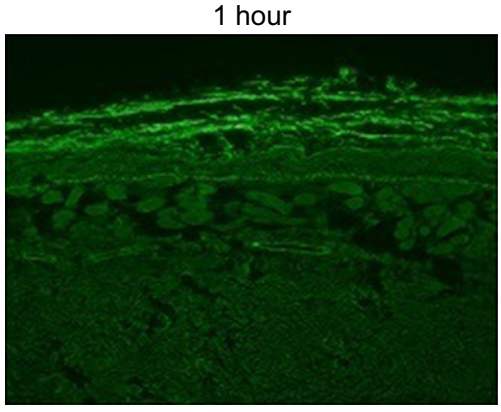

5 hours

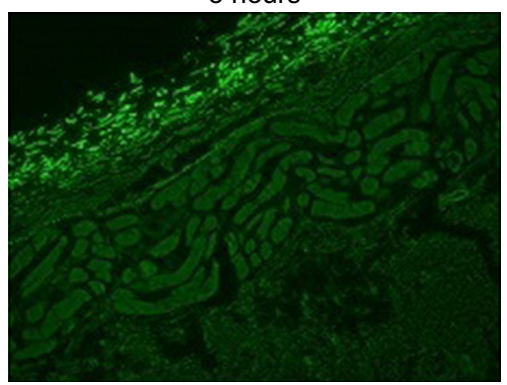

Figure 4 Fluorescence microscope images for rat skin layers following transdermal delivery of rhodamine florescence labeled NTF gel formula (F2) and raw (control) gel formula after I and 5 hours (magnification 400x).

Abbreviations: FIN, finasteride; NTF, nano-transferosomes.

with spherical shape and comparable average vesicle size range to the data obtained by using particle size analyzer. In addition, significant enhancement and improvement $(p<0.05)$ in the amount of FIN permeated across rat skin layers after 24 hours with 66.03, 48.98, and $41.81 \mu \mathrm{g}$ of FIN permeated from FIN-NTF gel formulations F1, F2, and F3, respectively, were achieved compared with raw FIN gel formula where the maximum amount of FIN permeated was $13.6 \pm 3.61 \mu \mathrm{g}$. This finding was confirmed with uniform fluorescence intensity of rhodamine-loaded NTF formula (F2) compared with raw rhodamine gel formula (control). The improved release of FIN from NTF gel formulations successfully indicated the possibility of FIN-NTF application as topical gel formula. According to these findings, FIN-NTF gel formulation could be a potential promising alternative drug delivery system that could possibly reduce FIN major side effects resulting from long-term oral administration for management of androgenetic alopecia.

\section{Acknowledgment}

This work was supported by the Deanship of Scientific Research (DSR), King Abdulaziz University, Jeddah (grant no D-155-166-1437). The authors, therefore, gratefully acknowledge the DSR's technical and financial support.

\section{Disclosure}

The authors report no conflicts of interest in this work.

\section{References}

1. Brenner S, Matz H. Improvement in androgenetic alopecia in 53-76-yearold men using oral finasteride. Int J Dermatol. 1999;38(12):928-930.

2. Hoffmann R. Finasteride is the main inhibitor of $5 \alpha$-reductase activity in microdissected dermal papillae of human hair follicles. Arch Dermatol Res. 1999;291(2-3):100-103.

3. Whiting D. Advances in the treatment of male androgenetic alopecia: a brief review of finasteride studies. Eur J Dermatology. 2001;11(4): 332-334.

4. Kaufman K. Finasteride, $1 \mathrm{mg}$ (Propecia), is the optimal dose for the treatment of men with male pattern hair loss. Arch Dermatol. 1999;135(8): 989-990.

5. D'Amico A, Roehrborn C. Effect of $1 \mathrm{mg} /$ day finasteride on concentrations of serum prostate-specific antigen in men with androgenic alopecia: a randomised controlled trial. Lancet Oncol. 2007;8(1):21-25.

6. Price V, Menefee E, Sanchez M. Changes in hair weight in men with androgenetic alopecia after treatment with finasteride (1 mg daily): three-and 4-year results. J Am Acad Dermatol. 2006;55(1):71-74.

7. Rathnayake D, Sinclair R. Male androgenetic alopecia. Expert Opin Pharmacother. 2010;11(8):1295-1304.

8. US Food and Drug Administration. Drug Approval Package Proscar (Finasteride) Tablets. Available from: https://www.accessdata.fda.gov/ drugsatfda_docs/nda/2003/020180_s027_ProscarTOC.cfm. Accessed December 14, 2017.

9. Hirshburg JM, Kelsey PA, Therrien CA, Gavino AC, Reichenberg JS. Adverse effects and safety of 5-alpha reductase inhibitors (finasteride, dutasteride): a systematic review. J Clin Aesthet Dermatol. 2016;9(7): $56-62$.

10. Carreño-Orellana N, Moll-Manzur C, Carrasco-Zuber JE, Álvarez-Véliz $\mathrm{S}$, Berroeta-Mauriziano D, Porras-Kusmanic N. Efectos adversos de finasteride: mitos y realidades. Una revisión actualizada [Finasteride adverse effects: An update]. Rev Med Chil. 2016;144(12):1584-1590. Spanish.

11. Caruso D, Abbiati F, Giatti S, et al. Patients treated for male pattern hair with finasteride show, after discontinuation of the drug, altered levels of neuroactive steroids in cerebrospinal fluid and plasma. $J$ steroid Biochem Mol Biol. 2015;146:74-79. 
12. Ahmed OAA, Afouna MI, El-Say KM, Abdel-Naim AB, Khedr A, Banjar ZM. Optimization of self-nanoemulsifying systems for the enhancement of in vivo hypoglycemic efficacy of glimepiride transdermal patches. Expert Opin Drug Deliv. 2014;11(7):1005-1013.

13. Caon T, Porto L, Granada A, et al. Chitosan-decorated polystyrene-bpoly (acrylic acid) polymersomes as novel carriers for topical delivery of finasteride. Eur J Pharm Sci. 2014;52:165-172.

14. Caserini M, Radicioni M, Leuratti C, Annoni O, Palmieri R. A novel finasteride $0.25 \%$ topical solution for androgenetic alopecia: pharmacokinetics and effects on plasma androgen levels in healthy male volunteers. Int J Clin Pharmacol Ther. 2014;52(10):842-849.

15. Maestrelli F, González-Rodríguez M. Effect of preparation technique on the properties of liposomes encapsulating ketoprofen-cyclodextrin complexes aimed for transdermal delivery. Int J Pharm. 2006;312(1-2): 53-60.

16. Mezei M, Gulasekharam V. Liposomes - a selective drug delivery system for the topical route of administration. Lotion dosage form. Life Sci. 1980;26(18):1473-1477.

17. Mezei M, Gulasekharam V. Liposomes - a selective drug delivery system for the topical route of administration: gel dosage form. JPharm Pharmacol. 1982;34(7):473-474.

18. Rajan R, Jose S, Mukund V. Transferosomes-A vesicular transdermal delivery system for enhanced drug permeation. J Adv Pharm Technol Res. 2011;2(3):138-143.

19. Badr-Eldin SM, Ahmed OAA. Optimized nano-transfersomal films for enhanced sildenafil citrate transdermal delivery: ex vivo and in vivo evaluation. Drug Des Devel Ther. 2016;10:1323-1333.

20. Cevc G, Blume G. New, highly efficient formulation of diclofenac for the topical, transdermal administration in ultradeformable drug carriers, Transfersomes. Biochim Biophys Acta. 2001;1514(2):191-205.

21. Benson HA. Transfersomes for transdermal drug delivery. Expert Opin Drug Deliv. 2006;3(6):727-737.

22. Bragagni M, Mennini N, Maestrelli F, Cirri M, Mura P. Comparative study of liposomes, transfersomes and ethosomes as carriers for improving topical delivery of celecoxib. Drug Deliv. 2012;19(7):354-361.
23. Mahmood S, Taher M, Mandal U. Experimental design and optimization of raloxifene hydrochloride loaded nanotransfersomes for transdermal application. Int J Nanomedicine. 2014;9:4331-4346.

24. Choi M, Maibach H. Elastic vesicles as topical/transdermal drug delivery systems. Int J Cosmet Sci. 2005;27(4):211-221.

25. Ntimenou V, Fahr A. Elastic vesicles for transdermal drug delivery of hydrophilic drugs: a comparison of important physicochemical characteristics of different vesicle types. J Biomed Nanotechnol. 2012;8(4): 613-623.

26. El-Nabarawi M, Bendas E, El Rehem R, MY A. Transdermal drug delivery of paroxetine through lipid-vesicular formulation to augment its bioavailability. Int J Pharm. 2013;443(1-2):307-317.

27. Shamma RN, Elsayed I. Transfersomal lyophilized gel of buspirone $\mathrm{HCl}$ : formulation, evaluation and statistical optimization. J Liposome Res. 2013;23(3):244-254.

28. Ahmed OA, Hussein AK, Mady FM. Optimisation of microstructured biodegradable finasteride formulation for depot parenteral application. J Microencapsul. 2016;33(3):229-238.

29. Ahmed OAA, Ahmed TA, Abdel-Naim AB, Khedr A, Banjar ZM, Afouna MI. Enhancement of in vitro skin transport and in vivo hypoglycemic efficacy of glimepiride transdermal patches. Trop J Pharm Res. 2014;13(8):1207-1213.

30. Higuchi T. Mechanism of sustained-action medication. Theoretical analysis of rate of release of solid drugs dispersed in solid matrices. J Pharm Sci. 1963;52(12):1145-1149.

31. Costa P, Sousa Lobo JM. Modeling and comparison of dissolution profiles. Eur J Pharm Sci. 2001;13(2):123-133.

32. El Zaafarany GGM, Awad GGAS, Holayel SSM, Mortada NDN. Role of edge activators and surface charge in developing ultradeformable vesicles with enhanced skin delivery. Int J Pharm. 2010;397(1-2): 164-172.

33. Benson H. Elastic liposomes for topical and transdermal drug delivery. Curr Drug Deliv. 2009;6(3):217-226.
Drug Design, Development and Therapy

\section{Publish your work in this journal}

Drug Design, Development and Therapy is an international, peerreviewed open-access journal that spans the spectrum of drug design and development through to clinical applications. Clinical outcomes, patient safety, and programs for the development and effective, safe, and sustained use of medicines are the features of the journal, which

\section{Dovepress}

has also been accepted for indexing on PubMed Central. The manuscript management system is completely online and includes a very quick and fair peer-review system, which is all easy to use. Visit http://www.dovepress.com/testimonials.php to read real quotes from published authors. 